\title{
Applying Research Metrics in Evaluating Online Learning
}

\author{
Khaiser Jahan Begum ${ }^{1}$ and Mohammad Bagher Negahban (1) 2,3,* \\ ${ }^{1}$ Department of Library and Information Sciences, University of Mysore, Manasagangothri, Mysuru, India \\ ${ }^{2}$ Shahid Bahonar University of Kerman, Kerman, Iran \\ ${ }^{3}$ Shiraz University of Medical Sciences, Shiraz, Iran \\ "Corresponding author: Assistant Professor of Information Sciences, Shahid Bahonar University of Kerman, Iran. Email: bm.negahban@gmail.com
}

Received 2019 May 23; Revised 2019 June 07; Accepted 2019 June 07.

Keywords: E-Learning, Research Metrics, Evaluation, Online

\section{Dear Editor,}

Training creative talents in the digital age is no longer restricted by geographical boundaries and this has been made possible through electronic and virtual learning (1, 2). Academic systems are now mandated with incorporating this new style of learning in their curriculums (3, 4). In this respect, higher education systems around the world have already started utilizing cybermetrics, webometrics, and scientometrics to pave the way for e-learning programs. The underlying question here is whether, in the light of recent developments in online education, it is possible to use research metrics as a modern means of evaluating electronic learning.

To answer this question, one should first note that educational evaluation may serve a wide range of different purposes. Some common purposes include a better understanding of learning objectives and expected outcomes by the end of learning programs, effectual support for these programs and activities, responsiveness at all stages of the learning process, better communication and outreach in education, etc.

Applying modern evaluation strategies will result in a more effective fulfillment of these objectives. On the one hand, it enriches teaching-learning interactions and enhances the learning experience in terms of efficiency and depth. On the other hand, it highlights the goals and expectations in educational environments. New evaluation methods also involve feedback analysis, which helps with managing the learning interactions, making the learning process a meaningful experience.

In studies that integrate teaching and learning indicators, scientometrics is applied to assess the level of communication and efficiency in teaching-learning activities of individual learners. In this respect, two studies were conducted with the aim of evaluating academic groups and the quality of education received by graduates in the United States (5). They were among the first major studies in which a combination of publication indices and teaching-learning indicators was applied. In addition to the bibliometric indicators, the authors used the indicators of the effectiveness of educational programs and educational quality.

Online learning can be evaluated through the qualitative assessment of technological competence using the indicators of technology diffusion and human skills. This assessment can be carried out by means of scientometrics and cybermetrics indicators.

Knowledge assessment methods and indicators can also be utilized to evaluate learning (6). These indicators are considered among the key factors in scientometric studies. Machine learning is heavily dependent on the results of the scientometric analysis. In this regard, using visualization techniques in the areas of knowledge and learning and scientific mapping based on the philosophy of drawing the structure of texts, which have for a long time been popular in scientometrics, are on high demand for learning assessment.

Therefore, online learning, as an innovative phenomenon, has turned into one of the most significant requirements of the modern age (7). At the same time, it should be noted that the nature and prospects of innovation are changing over time; hence, we need new assessment methods and indicators. Online learning is also a modern and innovative discipline closely intertwined with evaluation (8) and it is possible to evaluate it by means of research metrics. Applying these new methods will undoubtedly improve the quality of online learning. Using these principles and methods, we can achieve more qualitative results through measuring and evaluating the learning experience. The outcomes of these evaluations can 
then be applied to achieve various objectives of online learning, especially concerning its development and impact.

\section{Footnotes}

Authors' Contribution: Khaiser Jahan Begum developed the research methodology. Mohammad Bagher Negahban collected the required data, developed the statement of the problem, and reviewed the literature. Khaiser Jahan Begum and Mohammad Bagher Negahban authors contributed to the final revision of the manuscript.

Conflict of Interests: The authors declare that there is no conflict of interest in this study.

Ethical Approval: The study protocol was consistent with the ethical guidelines of the 1975 Declaration of Helsinki as reflected in a prior approval by the Institution's Human Research Committee.

Funding/Support: There was no support or funding for this research article.

\section{References}

1. Faraj Allah M, Haghighi F. Assessing the new strategic learning online for assessing online learning. Media. 2001;4(25-6).
2. Buchanan EA. Online assessment in high education: Strategies to systematically evaluate student learning. In: Howard C, Schunk K, Diseenza R, editors. Distance learning and university effectiveness: Changing educational paradigms for online learning. USA: Information Science Publishing; 2004. p. 163-76. doi: 10.4018/978-1-59140-178-0.ch008.

3. Means B, Toyama Y, Murphy R, Bakia M, Jones K. Evaluation of evidencebased practices in online learning a meta-analysis and review of online learning studies. Center for Technology in Learning; 2009. Available from: http://repository.alt.ac.uk/629/1/US_DepEdu_Final_report 2009.pdf.

4. Al-Jarf RS. The effects of web-based learning on struggling EFL college writers. Foreign Lang Ann. 2004;37(1):49-57. doi: 10.1111/j.19449720.2004.tb02172.x.

5. Beeckman D, Schoonhoven L, Boucque H, Van Maele G, Defloor T. Pressure ulcers: e-learning to improve classification by nurses and nursing students. J Clin Nurs. 2008;17(13):1697-707. doi: 10.1111/j.13652702.2007.02200.x. [PubMed: 18592624].

6. Chang MM. Enhancing web-based language learning through selfmonitoring. J Comput Assist Learn. 2007;23(3):187-96. doi: 10.1111/j.13652729.2006.00203.x

7. Tallent-Runnels MK, Thomas JA, Lan WY, Cooper S, Ahern TC, Shaw SM, et al. Teaching courses online: A review of the research. Rev Educ Res. 2016;76(1):93-135. doi: 10.3102/00346543076001093.

8. Karanjam SS, Zarif Sanaiey N, Karanjam S. Designing a comprehensive organizational e-learning model using the meta-synthesis method. Interdiscip J Virtual Learn Med Sci. 2017;In Press(In Press). doi: 10.5812/ijvlms.11496. 